\title{
Natural Background Levels in Groundwater
}

\author{
Elisabetta Preziosi ${ }^{1}$ (D) Marco Rotiroti ${ }^{2, *}$,, M. Teresa Condesso de Melo ${ }^{3}$ and Klaus Hinsby ${ }^{4}$ \\ 1 IRSA-CNR, Water Research Institute-National Research Council, Strada Provinciale 35d, 9, Montelibretti, \\ 00010 Rome, Italy; elisabetta.preziosi@cnr.it \\ 2 Department of Earth and Environmental Sciences, University of Milano-Bicocca, Piazza della Scienza 1, 20126 \\ Milano, Italy \\ 3 CERIS, Instituto Superior Técnico, University of Lisbon, Av. Rovisco Pais, 1049-001 Lisbon, Portugal; \\ teresa.melo@tecnico.ulisboa.pt \\ 4 Geological Survey of Denmark and Greenland (GEUS), Øster Voldgade 10, 1350 København K, Denmark; \\ khi@geus.dk \\ * Correspondence: marco.rotiroti@unimib.it
}

Citation: Preziosi, E.; Rotiroti, M.; Condesso de Melo, M.T.; Hinsby, K. Natural Background Levels in Groundwater. Water 2021, 13, 2770. https://doi.org/10.3390/w13192770

Received: 23 September 2021

Accepted: 5 October 2021

Published: 6 October 2021

Publisher's Note: MDPI stays neutral with regard to jurisdictional claims in published maps and institutional affiliations.

Copyright: (C) 2021 by the authors. Licensee MDPI, Basel, Switzerland. This article is an open access article distributed under the terms and conditions of the Creative Commons Attribution (CC BY) license (https:// creativecommons.org/licenses/by/ $4.0 /)$.
High levels of inorganic compounds in groundwater represent a significant problem in many parts of the world, with major economic, social and environmental drawbacks. The natural composition of groundwater derives mainly from the water-rock interactions, both in the vadose and saturated zone, but also depends on the biological and physicochemical processes, the residence time and the initial composition of the recharge water. Contamination from industrial, agricultural and urban areas often overlaps with the natural features of groundwater, and the assessment of the impact of anthropogenic activities might be challenging.

The distinction between natural and anthropogenic components that determine groundwater chemistry is a fundamental issue in groundwater management, particularly when the concentration of inorganic compounds exceeds the threshold values set for the evaluation of the good chemical status of groundwater bodies, as requested by many environmental regulations, including the European Water Framework and Groundwater Directives. In order to reach the good status of groundwater, natural background levels (NBLs) assessment of chemical species in groundwater is needed to clearly define the environmental objectives for groundwater bodies as well as the remediation targets for contaminated sites.

A variety of methodologies have been adopted by different countries to assess the NBLs of chemical species in groundwater that are potential pollutants and may be hazardous for human health and groundwater-dependent ecosystems (GDE). In Europe, the research efforts over the last 20 years have been dedicated to the elaboration of common standardized methodologies for the derivation of groundwater NBLs through the development of dedicated joint projects: from BASELINE [1] ("Natural Baseline Quality in European Aquifers: a basis for aquifer management") to BRIDGE [2] ("Background criteria for the identification of groundwater thresholds"), arriving to the ongoing project HOVER [3] ("Hydrogeological processes and Geological settings over Europe controlling dissolved geogenic and anthropogenic elements in groundwater of relevance to human health and the status of dependent ecosystems").

The continuous improvement in methodologies, tools and approaches for estimating groundwater NBLs has inspired this Special Issue, which aims to provide new insights into how NBLs are defined in different regions of the world, and to provide an update on the methods and approaches used to derive NBL at different spatial scales: site-specific, catchment, regional, national or transboundary scales.

The main goal of this Special Issue of Water is to bring together and discuss different methodological approaches and tools to improve the assessment of groundwater NBLs. From its first announcement, and after being thoroughly peer reviewed, nine papers have been accepted for publication [3-11]. To provide an overview of the experiences collected 
by this Special Issue, a brief summary of each published paper is reported below. A variety of spatial scales is encountered, ranging between national $[3,11]$, regional $[3,7,8,10]$, meso$[6,9]$ and site-specific $[4,5]$ scales, and different methodologies are applied.

For example, Lions et al. [3] describe the methodology developed within the EU project HOVER through its application to six study areas for the calculation of the NBLs of trace elements on a broad scale (regional or national). This method uses a statistical approach and is based on the lithological classification and on the land-use analysis, leading to the estimation of a NBL value for each class of lithology and geochemical conditions (e.g., $\mathrm{pH}$, redox) identified. The authors point out that this method provides consistent results when large datasets are available, and the aquifers have fairly homogeneous hydrogeological and hydrogeochemical conditions. Where local-scale variability is dominant or specific anthropogenic pressures are relevant, local investigations are needed. The work by Voutchkova et al. [11] compares the results of NBL estimation obtained from different HOVER and BRIDGE (preselection) applications for trace metals nationwide (Denmark). Pros and cons of the different methods used are highlighted. This allows the authors to propose a roadmap for NBL estimation at the national scale in countries with variable data availability. The roadmap provides "a systematic way of selecting an appropriate method or combination of methods to assure that NBLs are calculated based on groundwater data representing no or only very minor anthropogenic alterations to undisturbed conditions".

Another comparison of different methods is presented by Nakić et al. [8] for the estimation of the ambient background levels (ANLs) in four groundwater bodies in Croatia. The concept of ABL differs from that of NBL in that it addresses long-term human impacts (e.g., agriculture, urbanization) that have irreversibly altered the natural background hydrochemistry. The methods compared are (a) the BRIDGE preselection, (b) the probability plot and (c) the modified Lepeltier. Interestingly, the authors conclude by recommending the use of the probability plot and modified Lepeltier methods only when the censored data (i.e., concentrations below the limit of quantification) are less than $30 \%$, and the use of preselection with the censored data greater than $30 \%$.

Masciale et al. [7] and Parrone et al. [9] apply the Italian national guidelines for the assessment of the NBL in groundwater bodies, based on the preselection of data, enriched by temporal trend analyis and outliers treatment. The Italian Guidelines involve different procedures for the assessment of NBLs according to the spatial and temporal dimension of the sample and on the type of distribution of the pre-selected dataset, also considering the redox conditions of groundwater. The resulting NBLs are provided with their respective confidence levels, assessed on the basis of the total number of observations/monitoring sites, extent of the groundwater body and the type of aquifer. Both papers rely on a dedicated tool for the estimation of NBLs according to the national guidelines: eNaBLe [9]. The case studies are at the mesoscale (Vulsini volcanic district in central Italy) [9] and regional scale (Apulia region) [7].

Chidichimo et al. [5] also present a tool for calculating groundwater NBLs: GuEstNBL. This tool is able to assess groundwater NBLs by applying BRIDGE preselection and component separation methods. GuEstNBL is applied for the calculation of NBLs of trace elements on a site-specific scale (an industrial site located in Lamezia Terme, southern Italy). Here, the calculated NBLs support the definition of specific environmental cleanup goals.

Sacchi et al. [4] present another case of NBL assessment in groundwater on a sitescale case study: a former asbestos mine in Serpentinite in northern Italy. In mining environments, anthropogenic activities boost water-rock interactions, further increasing the concentration of potentially toxic elements, thus making the estimation of their groundwater NBL a challenging task. The authors use a statistical approach, based on the Italian Guidelines, to assess the NBLs in the former mining site. Interestingly, they conclude by giving a warning about the use of the median as a representative value for concentration time series of each monitoring station, as recommended by several guidelines (including BRIDGE); the median results in the elimination of half of the measurements and, 
in particular, higher concentration values, which could lead to overly conservative NBL estimates.

Filippini et al. [6] focus on the problem that national/regional groundwater monitoring networks may fail to capture hydrochemical heterogeneities on a local scale, possibly leading to misleading NBL values on a meso scale. In this work, the lack of information provided by the regional monitoring is filled with site-specific monitoring networks in contaminated/polluted sites under remediation. Since the common procedures for the assessment of NBLs cannot be applied to this type of dataset due to the limited homogeneity of the data, an "unorthodox" method is proposed based on the definition of a consistent working dataset, followed by a statistical identification and a critical analysis of the outliers.

Finally, Shahzad et al. [10] report a case study analysing the spatial (regional) and temporal variability of groundwater quality data using statistical and geostatistical techniques. Although NBLs are not calculated, this work highlights the importance of exploratory analysis in supporting the definition of hydrogeochemical conceptual models, further supporting the assessement of groundwater NBLs.

The articles presented in this Special Issue provide an overview of the different approaches and protocols in use for NBL assessment, ranging from well-known methods such as component separation or cumulative probability plot methods, to new computeraided protocols or the recent "HOVER methodology" [3], based on the exploitation of large datasets in regions where groundwater monitoring networks have been operating for many years.

A variety of case studies describe different situations where NBL is needed to properly assign good or poor quality status to groundwater bodies. Key issues are highlighted, such as the correct definition of conceptual models, the necessity to clearly differentiate between pre-selection criteria and thresholds for NBL definition, the link between NBL assessment and groundwater monitoring networks and the need for targeted analytical methods to achieve lower LOQs.

Current trends in NBL assessment focus on methods for assessing the spatial distribution and temporal variation of NBLs that could impact the legal assessment of polluted sites or water bodies. Overall, this collection of papers will be a source of inspiration for future research and development on this topic. It is not yet possible to make a recommendation for a preferred method to derive NBLs, which would be robust enough to be applied in most hydrogeological and land use settings across Europe and worldwide, providing comparable NBLs. The selection of published articles provides examples covering a range of different settings that will be relevant to many, but not all settings commonly found in Europe and worldwide. In all cases, an individual assessment is required to decide whether one or more of the methods presented in this Special Issue will meet the needs of NBL derivation in other settings.

Author Contributions: Writing—original draft, E.P. and M.R.; writing—review and editing, E.P., M.R., M.T.C.d.M. and K.H. All authors have read and agreed to the published version of the manuscript.

Funding: This research received no external funding.

Acknowledgments: The authors appreciate the efforts of the Water editors and the publication team at MDPI, and the anonymous reviewers for their invaluable comments.

Conflicts of Interest: The authors declare no conflict of interest.

\section{References}

1. Nieto, P.; Custodio, E.; Manzano, M. Baseline Groundwater Quality: A European Approach. Environ. Sci. Policy 2005, 8, 399-409. [CrossRef]

2. Hinsby, K.; Condesso de Melo, M.T.; Dahl, M. European Case Studies Supporting the Derivation of Natural Background Levels and Groundwater Threshold Values for the Protection of Dependent Ecosystems and Human Health. Sci. Total Environ. 2008, 401, 1-20. [CrossRef] [PubMed] 
3. Lions, J.; Devau, N.; Elster, D.; Voutchkova, D.D.; Hansen, B.; Schullehner, J.; Petrović, P.T.; Samolov, K.A.; Camps, V.; Arnó, G.; et al. A Broad-Scale Method for Estimating Natural Background Levels of Dissolved Components in Groundwater Based on Lithology and Anthropogenic Pressure. Water 2021, 13, 1531. [CrossRef]

4. Sacchi, E.; Bergamini, M.; Lazzari, E.; Musacchio, A.; Mor, J.-R.; Pugliaro, E. Natural Background Levels of Potentially Toxic Elements in Groundwater from a Former Asbestos Mine in Serpentinite (Balangero, North Italy). Water 2021, 13, 735. [CrossRef]

5. Chidichimo, F.; Biase, M.D.; Costabile, A.; Cuiuli, E.; Reillo, O.; Migliorino, C.; Treccosti, I.; Straface, S. GuEstNBL: The Software for the Guided Estimation of the Natural Background Levels of the Aquifers. Water 2020, 12, 2728. [CrossRef]

6. Filippini, M.; Zanotti, C.; Bonomi, T.; Sacchetti, V.G.; Amorosi, A.; Dinelli, E.; Rotiroti, M. Deriving Natural Background Levels of Arsenic at the Meso-Scale Using Site-Specific Datasets: An Unorthodox Method. Water 2021, 13, 452. [CrossRef]

7. Masciale, R.; Amalfitano, S.; Frollini, E.; Ghergo, S.; Melita, M.; Parrone, D.; Preziosi, E.; Vurro, M.; Zoppini, A.; Passarella, G. Assessing Natural Background Levels in the Groundwater Bodies of the Apulia Region (Southern Italy). Water 2021, $13,958$. [CrossRef]

8. Nakić, Z.; Kovač, Z.; Parlov, J.; Perković, D. Ambient Background Values of Selected Chemical Substances in Four Groundwater Bodies in the Pannonian Region of Croatia. Water 2020, 12, 2671. [CrossRef]

9. Parrone, D.; Frollini, E.; Preziosi, E.; Ghergo, S. eNaBLe, an On-Line Tool to Evaluate Natural Background Levels in Groundwater Bodies. Water 2021, 13, 74. [CrossRef]

10. Shahzad, H.; Farid, H.U.; Khan, Z.M.; Anjum, M.N.; Ahmad, I.; Chen, X.; Sakindar, P.; Mubeen, M.; Ahmad, M.; Gulakhmadov, A. An Integrated Use of GIS, Geostatistical and Map Overlay Techniques for Spatio-Temporal Variability Analysis of Groundwater Quality and Level in the Punjab Province of Pakistan, South Asia. Water 2020, 12, 3555. [CrossRef]

11. Voutchkova, D.D.; Ernstsen, V.; Schullehner, J.; Hinsby, K.; Thorling, L.; Hansen, B. Roadmap for Determining Natural Background Levels of Trace Metals in Groundwater. Water 2021, 13, 1267. [CrossRef] 